\title{
Review: medication and cognitive behaviour therapy control symptoms of bulimia nervosa
}

\author{
Whittal ML, Agras WS, Gould RA. Bulimia nervosa: a meta-analysis of psychosocial and pharmacological treatments. Behavior \\ Therapy 1999 Winter;30:117-35.
}

\section{Question}

In people with bulimia nervosa, how do medication and cognitive behaviour therapy (CBT) compare in controlling binge and purge frequency, depression, and eating attitudes?

\section{Data sources}

Studies were identified by searching Medline (1966-98) and PsycLIT (1974-98) using the terms bulimia, bulimia nervosa, treatment outcome, clinical trial, and double blind; references of studies were reviewed; and journals specific to eating disorders were searched for the previous 8 years.

\section{Study selection}

Studies were selected if they were randomised controlled trials of patients diagnosed with bulimia nervosa according to DSM-III criteria. Studies of pharmacological interventions had to be double blind and placebo controlled. Psychosocial studies could involve CBT, behaviour therapy, or exposure and response prevention, but for the purposes of this review, all were referred to as CBT.

\section{Data extraction}

Data were extracted on number of patients, treatment type and duration, and proportion of patients who dropped out or were abstinent after treatment. Pooled effect sizes were calculated for studies with no significant heterogeneity to assess the magnitude of change from before treatment to after treatment for 4 outcomes: binge frequency, purge frequency, depression, and eating attitudes. Positive effect sizes indicated improvement in symptoms.

\section{Main results}

9 medication trials (870 patients) and 26 CBT trials (460 patients) were included. Medication and CBT were effective for all 4 outcomes (table). For each outcome the effect sizes for CBT were higher than for medication. Combined treatment was effective for binge and purge frequency (table). Heterogeneity existed among combined treatment studies for depression and eating attitudes. Combined treatment was more effective than medication alone for binge and purge frequency and more effective than CBT alone for binge frequency. The dropout rates for medication trials and CBT trials did not differ.

\section{Conclusions}

In people with bulimia nervosa, medication and cognitive behaviour therapy are both effective in controlling binge and purge frequency, depression, and eating attitudes. Cognitive behaviour therapy is the most effective single treatment.

\begin{tabular}{lll}
\hline & $\begin{array}{l}\text { Number of studies } \\
\text { with no significant } \\
\text { heterogeneity }\end{array}$ & $\begin{array}{l}\text { Overall weighted effect } \\
\text { size }(95 \% \text { CI })\end{array}$ \\
\hline Medication trials & & \\
$\quad$ Binge frequency & 9 & $0.66(0.52$ to 0.81$)$ \\
Purge frequency & 6 & $0.39(0.24$ to 0.54$)$ \\
Depression & 9 & $0.73(0.58$ to 0.88$)$ \\
$\quad$ Eating attitudes & 8 & $0.71(0.56$ to 0.86$)$ \\
CBT trials & 17 & $1.28(1.09$ to 1.47$)$ \\
Binge frequency & 24 & $1.22(1.06$ to 1.39$)$ \\
Purge frequency & 19 & $1.31(1.10$ to 1.51$)$ \\
$\quad$ Depression & 13 & $1.35(1.12$ to 1.58$)$ \\
Eating attitudes & & $1.77(1.34$ to 2.21$)$ \\
Combined treatment & 4 & $1.33(0.94$ to 1.73$)$ \\
$\quad$ Binge frequency & 5 & \\
Purge frequency & &
\end{tabular}

Source of funding: no external funding.

For correspondence: $\operatorname{Dr} M L$ Whittal, Vancouver Hospital and Health Sciences Centre-UBC site, 2211 Wesbrook Mall, Vancouver, BC V6T 2B5, Canada. Fax +1 6048227452

A modified version of this abstract appears in Evidence-Based Mental Health and Evidence-Based Medicine.

\section{Commentary}

Assisting people to recover from bulimia nervosa requires that nurse clinicians are knowledgeable about available treatments.The meta-analysis by Whittal et al assesses the effectiveness of medications and CBT using larger sample sizes than individual studies permit. The study results are consistent with previous findings recommending $\mathrm{CBT}$ as the treatment of choice for bulimia nervosa. ${ }^{1}$ The suggestion that a combination of CBT plus antidepressants may be more effective than CBT alone warrants further research.

The exclusive use of randomised studies, a thorough search to locate relevant studies, and an attempt to obtain unreported data (eg, means and SDs) from authors sets a positive example for students and frontline workers who want unequivocal information about the topic.
To address publication bias (the greater likelihood for studies with significant findings to be published ${ }^{2}$ ), the authors statistically determined the number of unpublished studies with non-significant findings that would be needed to change the findings of their meta-analyses.

The authors did not report criteria for assessing the methodological quality of the primary studies. Descriptions of sample characteristics (eg, age, sex, and ethnicity) would have helped readers to determine whether the study populations were similar to their own patients. Whittal et al used a controversial method to analyse the data by excluding studies with effect sizes that were most discrepant from the average until a significant test for heterogeneity became non-significant. Some would argue that exclusion of these studies could result in a biased overall effect size.
Nevertheless, the results of this study are relevant to nurse clinicians who work with bulimia in any setting and will compel me to examine and refine more closely the CBT content of my approach to these patients. Further studies weighing expected benefits against potential harms and costs and those comparing specific treatment variables (eg, duration, format, and individual components of CBT) are needed to inform practice.

Kathy Weaver, RN, BN Instructor, Faculty of Nursing University of New Brunswick Fredericton, New Brunswick, Canada

1 Garner DM, Rockert W, Davis R, et al. Comparison of cognitive-behavioural and supportive-expres supportive-expressive therapy for bulimis

nervosa. Am J Psychiatry 1993;150:37-46.

Wolf FM. Meta-analysis: quantitative methods for research synthesis. Quantitative applications in the social sciences, Series/Number 07-059. 